Experience with Gestrinone Therapy in the Infertile Patient (Mild Endometriosis)

B. Hedon, V. Sautecoeur, J.M. Chinchole, B. Galand, G. Imbernon, S. Neveu, P. Mares, F. Laffargue, J.L. Viala

Services de Gynécologie-Obstétrique, Montpellier, France

During a one-year period (1985) 40 consecutive infertile patients for which mild endometriosis was diagnosed by laparoscopy were treated with gestrinone (Roussel-Uclaf, Paris, France), $2.5 \mathrm{mg}$ twice a week, during 4 months. All these patients had infertility of long duration (57 months). Infertility could not be sufficiently explained in view of the results of the basic work-up, either that this basic work-up was totally normal, or that anomalies exhibited were not sharp enough to be the only probable reason for the infertile condition.

Gestrinone therapy was well tolerated in the majority of the cases. The main discomfort was due to frequent bleeding (24\%). Four spontaneous pregnancies were obtained with a main delay between the end of therapy and the beginning of pregnancy of 8 months. Two more pregnancies were obtained by in vitro fertilization (13 cycles, 11 patients, 2 pregnancies).

Conclusions are that gestrinone is a well-tolerated therapy, but its efficacy on endometrial implants and on subsequent infertility associated with endometriosis remains to be confirmed.

Effects of Buserelin Administration on the Androgenic Profile

L. Barrel, J. Calaf, A. Cabero, P. Viscasillas, J. Rodriguez Espinosa Hospital de Sant Pau, Barcelona, Spain

The administration of a gonadotropin-releasing hormone superagonist enables the depression of ovarian function through the neutralization and down-regulation of gonadotroph membrane receptors and consequent impairment of pituitary gonadotropin synthesis and secretion. When follicular development is restricted to the early stages, the tecal structures secrete predominantly androgens.

On the other hand, since estrogens are diminished, sex hormone binding globulin (SHBG) secretion at hepatic levels could also be impaired, thus resulting in increased levels of free androgens.

All this could result in the existence of a predominantly androgenic hormonal profile in the women treated with this substance. To study this aspect, 12 normally cycling women, being treated for endometriosis with buserelin (HOE 766) at doses of $300 \mathrm{pg}$ 
every $8 \mathrm{~h}$ intranasally, for 6 months, have been considered. Basal, 15-day and afterwards monthly serum samples were taken and kept frozen until the end of the study. Luteinizing hormone $(\mathrm{LH})$, estradiol testosterone and SHBG were determined by radioimmunoassay and a free testosterone index $(\mathrm{FTI})$ has been calculated. $\mathrm{FTI}=$ (T/SHBG) X 100.

\section{Posters 159}

A variance analysis has shown a significant decrease in estradiol levels, while testosterone, LH, SHBG, and FTI levels did not experience any significant change.

According to these findings it can be concluded that the chronic ovarian suppression with buserelin did not influence negatively the androgenic profile.

Laparoscopic Explorative Cyto-Puncture and Aspiration of Intra-Ovarian Endometriotic Cysts and Deposits 186

Cases in 22 Years

Michel Mintz

Paris, France

$84 \%$ of the patients are between 20 and 40 years old, $70 \%$ are nulliparous or sterile. Among 214 punctured cysts, $62 \%$ contained between 10 and $400 \mathrm{ml}$ (27-91 mm diameter). Judged by 79 surgical controls, the reliability of cytology is $84 \%$ : it eliminated corpus luteum cysts, revealed among 214 ovarian endometriosis almost $50 \%$ of intra-ovarian hidden cases, and contributed to the discovery of 3 endometriotic malign cysts of benign aspect ( 1 false-negative).

Among 186 patients, 24 had complementary surgical cyst resections. 139 patients bearing 155 cysts were followed-up after puncture: $80 \%$ for more than 2 years, $50 \%$ between 6 and 20 years. Most of the prescriptions (33 different gynecologists) were not correctly followed.

Among 109 second-looks and/or ultrasonic controls: 47 cases of persistance or recurrence, 62 showed no cysts, although 15 had revealed cysts from 50 to $100 \mathrm{ml}$. Among 65 patients who wanted children, 31 bore 40 known living children, although 16 had had large punctured cysts of 50-400 ml.

\section{Conclusions}

This technique seems appropriate to young, nulliparous or sterile women, and should be used before long prescriptions and before surgical resections which do not always prevent recurrence and may cause adhesions.

For elder patients, surgical resection of endometriotic cysts should be performed, since it concerns a risk group of ovarian cancer. 
Attila Török, Jôzsef Bôdis, Imre Csaba

Department of Obstetrics and Gynecology, University Medical School of Pécs, Pécs, Hungary

Fifteen patients were treated with $400 \mathrm{mg} / \mathrm{day}$ and another 15 patients with 300 $\mathrm{mg} / \mathrm{day}$ of danol. Blood samples were taken before the treatment and in monthly intervals thereafter. Serum luteinizing hormone ( $\mathrm{LH})$, follicle-stimulating hormone $(\mathrm{FSH})$, prolactin, hydroxycortisone, estradiol and progesterone levels were determined with radioimmunoassays. 